\title{
Response of an oscillating superleak transducer to a pointlike heat source
}

\author{
A. Quadt, B. Schröder, M. Uhrmacher,* J. Weingarten, B. Willenberg, and H. Vennekate \\ Universität Göttingen, II. Physikalisches Institut, Friedrich-Hund-Platz 1, 37077 Göttingen, Germany
}

(Received 11 November 2011; published 1 March 2012)

\begin{abstract}
A new technique of superconducting cavity diagnostics has been introduced by D. L. Hartill at Cornell University, Ithaca, New York. It uses oscillating superleak transducers (OST) which detect the heat transferred from a cavity's quench point via Second Sound through the superfluid He bath, needed to cool the superconducting cavity. The localization of the quench point is done by triangulation. The observed response of an OST is a nontrivial, but reproducible pattern of oscillations. A small helium evaporation cryostat was built which allows the investigation of the response of an OST in greater detail. The distance between a pointlike electrical heater and the OST can be varied. The OST can be mounted either parallel or perpendicular to the plate that houses the heat source. If the artificial quench point releases an amount of energy compatible to a real quench spot on a cavity's surface, the OST signal starts with a negative pulse, which is usually strong enough to allow automatic detection. Furthermore, the reflection of the Second Sound on the wall is observed. A reflection coefficient $R=0.39 \pm 0.05$ of the glass wall is measured. This excludes a strong influence of multiple reflections in the complex OST response. Fourier analyses show three main frequencies, found in all OST spectra. They can be interpreted as modes of an oscillating circular membrane.
\end{abstract}

DOI: 10.1103/PhysRevSTAB.15.031001

\section{INTRODUCTION}

In modern particle accelerators the cavities are generally made of superconducting materials with a high critical temperature, like niobium with $T_{c}=9.2 \mathrm{~K}$. At the low operating temperature of a cavity (1.5-4 K), the Ohmic losses are reduced and almost all the rf power from the klystrons is available to accelerate the beam. Quality factors of $Q_{0}=10^{9}-10^{10}$ are obtained. Niobium can be sputtered on the interior surface of a copper cavity as for those in the LHC, or the whole cavity can be made from ultrapure niobium. A typical example of the latter is the TESLA cavity [1,2] which consists of 9 cells, has a total length of about $1 \mathrm{~m}$, and is operated at $1.3 \mathrm{GHz}$. These cavities are used at FLASH and soon at the X-ray Free Electron Laser (XFEL), both free electron lasers at DESY, and they are being considered for the future International Linear Collider (ILC) collider.

The niobium coated copper cells of the LHC produce an accelerating electric field gradient of $5 \mathrm{MV} / \mathrm{m}$, while the TESLA cavities were designed to run at a gradient of at least $15 \mathrm{MV} / \mathrm{m}$. They should reach $23.6 \mathrm{MV} / \mathrm{m}$ at the $\mathrm{XFEL}$ and $31.5 \mathrm{MV} / \mathrm{m}$ for the ILC [3,4]. Still higher gradients would reduce the number of cavities needed and lower the costs. The maximum gradient is limited by the critical magnetic field $H_{c}$, niobium with $H_{c} \sim 200 \mathrm{mT}$

\footnotetext{
*Michael.Uhrmacher@gmx.de
}

Published by the American Physical Society under the terms of the Creative Commons Attribution 3.0 License. Further distribution of this work must maintain attribution to the author(s) and the published article's title, journal citation, and DOI.
PACS numbers: 03.75.Kk, 07.20.Mc, 43.38.+n, 67.25.dm

at $2 \mathrm{~K}$ is probably usable for gradients up to $E_{\text {acc }} \sim$ 55-60 MV/m [5].

There standard problems are encountered while trying to reach these high gradients. Electrons and discharges are produced inside the cavity, which often start at imperfections of the inner walls. Heat is generated at these spots, the superconductivity is lost and still more heat occurs due to resistive heating: the cavity quenches and the stored energy is released. During the course of cavity development several different treatments are used to optimize the inner surfaces of the cavities in order to avoid this (e.g. grinding, high pressure water rinsing, heat treatment, buffered chemical or electropolishing, etc.) [2].

Each cavity has to be tested before it is inserted into a larger accelerator facility. The present $500 \mathrm{GeV}$ ILC design requires about 18000 TESLA niobium cavities (including spares) [6]. The classical way of testing a cavity for quench spots is the use of arrays of thermocouples, which are moved around the cavity in operation. Quench spots can be localized to within 2-3 cm, but "the process of locating defects in 9 cell cavities remains a lengthy and cumbersome process" [7]. Practically, it cannot be applied on the thousands of ILC cavities.

In 2008, a new technique was introduced at Cornell University to trace the position of a quench spot [8,9]. The principle of the method is the following: When a superconducting cavity is operated below $2.17 \mathrm{~K}$, the liquid $\mathrm{He}$ in the cooling container becomes superfluid (He-II). If the cavity cannot stand the accelerating gradient, a quench occurs. The temperature at the quench point rises and generates a "Second Sound" wave which travels through the liquid He-II. The oscillating superleak transducer 
(OST) can be used to track such a wave in this environment. The time interval between the quench and the arrival of the Second Sound at an OST can be measured, typically $0.5 \mathrm{~ms}$ per $\mathrm{cm}$ distance. The application of multiple OSTs at selected positions allows for a localization of the quench spot via triangulation.

The signal of an OST is a complex superposition of the Second Sound wave and the eigenmodes of the OST membrane. Further complication of the signal shape can occur due to Second Sound waves reflected at the cavity walls, the walls of the cryostat, etc. To disentangle these effects, the response of an OST is studied in the simplified environment of a small He evaporation glass cryostat.

\section{SECOND SOUND IN SUPERFLUID HELIUM}

\section{A. Properties of liquid $\mathrm{He}$}

Cavities in a modern accelerator are placed inside a cooling bath of liquid He. The phase diagram (Fig. 1) shows that at normal pressure $(100 \mathrm{kPa})$ liquid $\mathrm{He}$ has a temperature of $4.22 \mathrm{~K}$. If the pressure is reduced, He cools down to the triple point $T_{\lambda}=2.17 \mathrm{~K}$ at the pressure of $p_{\lambda}=4.9 \mathrm{kPa}$ [10]. Below $T_{\lambda}$ liquid He undergoes a transition into a superfluid phase (He-II), where it has no internal friction and nearly perfect heat conductivity.

A phenomenological description of this transition was first given by Tisza and later by Landau [11]. They suggested an ideal mixture of a normal fluid and a superfluid below $T=2.17 \mathrm{~K}$. The normal fluid has density $\rho_{n}$ and viscosity $\eta_{n}$. It travels with the velocity $v_{n}$ and carries the total entropy $S$ of the system. The superfluid has the density $\rho_{s}$, a viscosity $\eta_{s}=0$, a vanishing entropy and

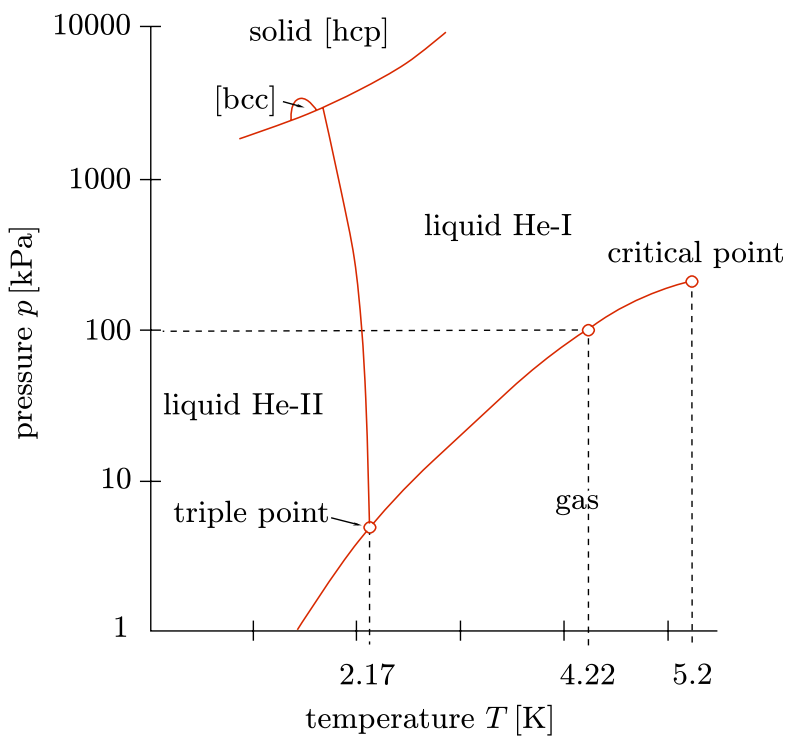

FIG. 1. Helium phase diagram [10]. Cooling down helium under normal pressure the liquid phase occurs at $4.2 \mathrm{~K}$. Reduction of the pressure to $49 \mathrm{hPa}$ cools the liquid to the triple point where superfluidity occurs.

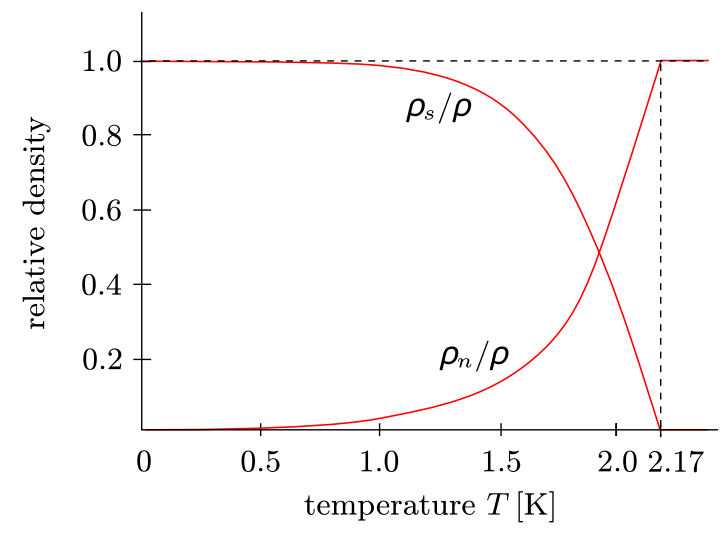

FIG. 2. Relative density versus temperature below the $\lambda$ point in helium.

travels with the velocity $v_{s}$. The total density is the sum of the two components: $\rho=\rho_{s}+\rho_{n}$.

As shown in Fig. 2 the density ratio is strongly temperature dependent between 1.0 and $2.17 \mathrm{~K}$. One can define the mass flow of He below the $\lambda$ point to be $j=\rho_{s} v_{s}+\rho_{n} v_{n}$. As shown in $[12,13]$, two types of heat transport can be derived in liquid He below the $\lambda$ point: The First Sound, a pressure wave with velocity $v_{\mathrm{FiSo}}$, and the Second Sound, an entropy wave with velocity $v_{\text {SeSo }}$.

\section{B. Second sound}

While the First Sound is a longitudinal fluctuation in density driven by pressure, the Second Sound is a temperature driven wave, opposite in phase movement of the normal and superfluid components; it is an entropy wave that transports heat. The velocity $v_{\text {SeSo }}$ of this heat transfer has a strong temperature dependence, as can be deduced from the temperature dependence of the density ratios given in Fig. 2. Various measurements were performed to determine $v_{\text {SeSo }}$.

Figure 3 shows data from the measurement of $v_{\text {SeSo }}$ performed by Wang et al. [14] in resonance experiments. The blue curve is a polynomial fit to the data of [14]. During the experiments presented here, the temperature is kept between 1.5 to $1.8 \mathrm{~K}$, where the velocity of the

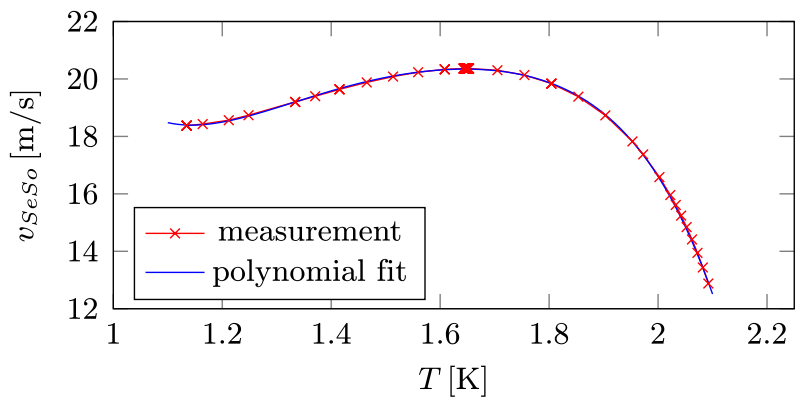

FIG. 3. Second Sound velocity $v_{\text {SeSo }}$ versus temperature [14]. 
Second Sound is nearly constant at a value of $v_{\text {SeSo }} \sim$ $20 \mathrm{~m} / \mathrm{s}=2 \mathrm{~cm} / \mathrm{ms}$. Nevertheless, the actual velocity is derived from the polynomial fit to Wang 's [14] data and the measured temperature of the He.

\section{SECOND SOUND RAY TRACING AROUND A TESLA CAVITY-A SIMULATION}

\section{A. Simplifying assumptions}

The Second Sound localization technique is studied using simulations of the TESLA 9-cell cavity. The length of the whole structure is $1276 \mathrm{~mm}$, while the single cells are $115.4 \mathrm{~mm}$ long and $206 \mathrm{~mm}$ wide [1]. The diameter of the iris diaphragms between the cells is $70 \mathrm{~mm}$. In the simulation program the shape is simplified to a central cylinder and nine half tori, which cover the critical regions of the cavity well. All additional parts like high order mode couplers are neglected. The critical region is the equator of each cell where two half cells are welded together. The resulting welding seam is the main source for material disorder. Furthermore, it coincides with the region of highest magnetic field. Therefore, for the simulation, the quench spots are randomly placed around the equators of the cells.

The placement of the OSTs is limited by the geometry of a liquid He tank, used for testing TESLA cavities. To allow for a fast cavity exchange during testing, one plane with OSTs can be mounted permanently on the bottom of the tank, the others can be mounted on the cover of the tank. The distance between OST and equator is assumed to be $103 \mathrm{~mm}$, i.e., on a circle with twice the maximum radius of the cavity.

A constant velocity of the Second Sound is assumed $(v=20 \mathrm{~m} / \mathrm{s}$ ) in the simulations. For the reconstruction only lines of direct sight are used. As a consequence, every quench above a cells equator should not be seen by a bottom detector and vice versa. According to the wave nature of Second Sound, events within a distance of less than $20 \mathrm{~mm}$ distance to the cell's equator are propagated around the equator to be registered by an OST on the other side. It was shown that two planes with six detectors are sufficient in the selected geometry to have always two OSTs with direct lines of sight to the quench spot. Neither reflections nor attenuations are implemented. Details of the simulations can be found in [15].

\section{B. Results and consequences}

The first results of the simulations show that quenches are in general detected by two or three OSTs with direct lines of sight, in the case of six detectors on each of the two levels (on the top and bottom of the cryostat). Two-signal events, which are predominantly observed for quenches in the central cells, can be divided in two subgroups depending on whether the quench is observed by OSTs on the same detector plane or not. (a) When the two firing OSTs

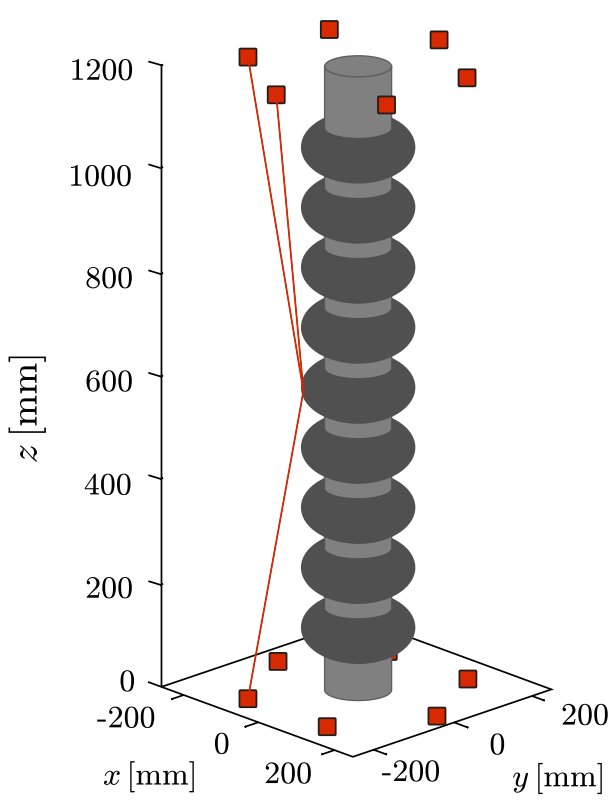

FIG. 4. TESLA cavity in the simulation with $2 \times 6$ OSTs (red squares). A three signal event is shown.

sit on the same plane the reconstruction is straightforward. (b) The critical case is a signal in two OSTs in different planes (see Fig. 4 and take only one red line in the upper part). The reconstruction will give two possible quench locations. In most cases, one of the proposed locations is within a few millimeters at the original quench spot. The mean deviation between a real spot and the reconstructed one is about $5.7 \mathrm{~mm}$.

In the case of three or more responding OSTs (see Fig. 4), the spot is unambiguously localized with a mean deviation of only $4.5 \mathrm{~mm}$. A third plane of OSTs in the middle of the cryostat, i.e., of the cavity, would increase the localization precision. The simulations show that it is possible to cover nearly $99 \%$ of the critical regions of a TESLA cavity with twelve detectors on two levels, six at the top and six at the bottom of the cryostat [15]. Nevertheless, the geometry of a real cavity is more complex as additional big parts might be inside the He tank. The question arises whether strong reflections of the Second Sound on the surfaces occur. These would increase the complexity of the quench-localization algorithms.

\section{EXPERIMENTS INSIDE A HELIUM EVAPORATION CRYOSTAT}

\section{A. The apparatus}

A He evaporation cryostat was built as a test volume for Second Sound waves, which are produced by a tiny heat source and measured by an OST which is mounted on a movable support rod [16]. The cryostat consists of two nested Dewars. The outer one contains liquid $\mathrm{N}_{2}$ as a cooling shield for the inner Dewar which is filled with liquid He. The hollow glass walls are evacuated and 
mirrored to provide a high degree of heat insulation. The inner He Dewar is closed with a stainless steel chamber, which houses all necessary connections (electrical for OST and heater, mechanical for vacuum pumping, pressure measurements, a feedthrough for the OST mount, and one for filling the liquid $\mathrm{He}$ into the inner Dewar). The heat source (see Sec. IV C) is positioned on the central axis close to the bottom of the Dewar. The OST rod is pivoted and can be positioned at any height in the He Dewar. The rod axis is shifted by $12.5 \mathrm{~mm}$ with respect to the central axis of the $70 \mathrm{~mm}$ wide Dewar to allow for noncentral positioning of the OST, by turning. Figure 5 shows the setup. A rotary vane pump (Alcatel 2033 with $30 \mathrm{~m}^{3} / \mathrm{h}$ suction power) is used to lower the pressure in the gas volume above the surface of the liquid He. Evaporation cools the liquid to 1.5 or $1.8 \mathrm{~K}$ (approximately $5-17 \mathrm{hPa}$ ) into the superfluid He phase. By pumping out the He vapor, the total amount of $\mathrm{He}$ in the Dewar is reduced over time. A maximum distance between the heat source and the OST of about $44 \mathrm{~cm}$ can be realized at the beginning of the measurements. After 8 hours the liquid $\mathrm{He}$ level is at $20 \mathrm{~cm}$. The temperature is controlled by the pressure of the gaseous He according to the International Temperature Scale (ITS-90) [17]. The precision of the temperature determination is obtained from the pressure measurement. In the experiment a logarithmic membrane vacuum meter is used (DIAVAC DV1000, Oerlikon Leybold Vacuum), connected by a steel bellow to the cryostat's head. The

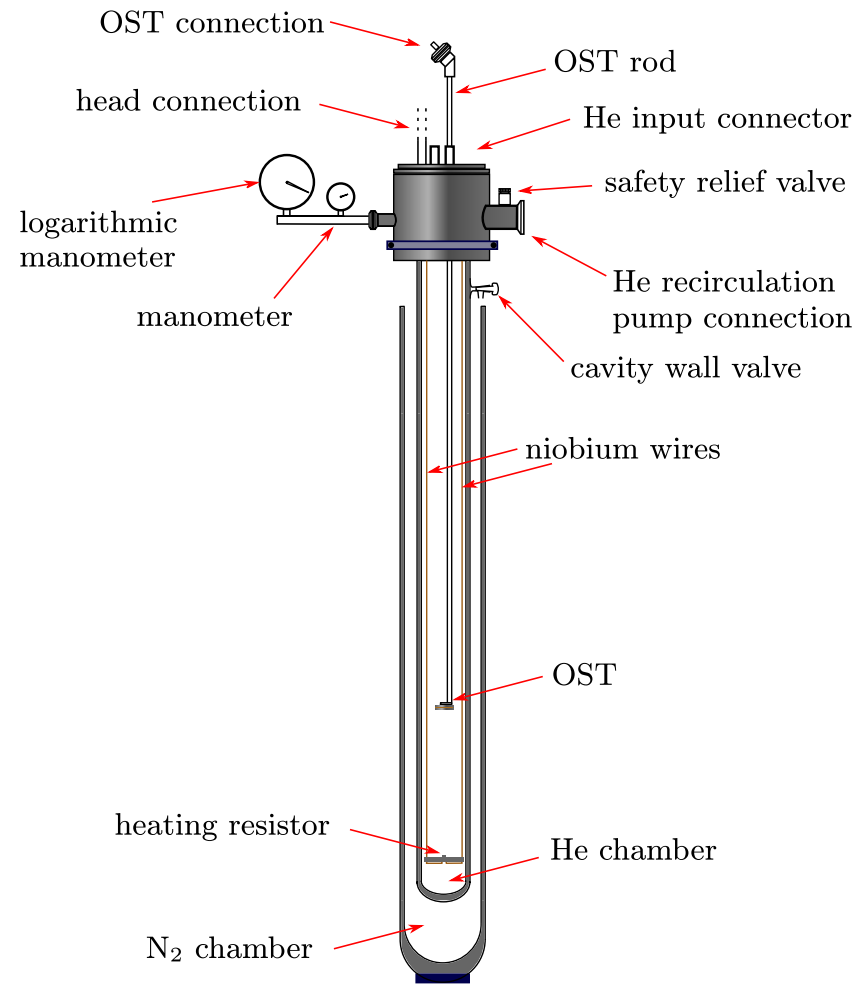

FIG. 5. Sketch of the He evaporation cryostat with mounted OST and heat source. absolute accuracy in the pressure range of 1 to $10 \mathrm{hPa}$ is $\pm 1 \mathrm{hPa}$ [18]. This corresponds to an error on the temperature of $\Delta T= \pm 0.05 \mathrm{~K}$.

\section{B. OST-Design and working principle}

Oscillating superleak transducers (OST) can be used for excitation and detection of Second Sound in superfluid helium. The concept is similar to a condenser microphone. The functional part is a membrane ( $M$ in Fig. 6, active diameter: $19.6 \mathrm{~mm}$ ) made from a cellulose nitrate membrane filter of $0.1 \mathrm{~mm}$ thickness (made by VWR Scientific, USA) which contains pores with a diameter of $0.2 \mu \mathrm{m}$ [16].

These pores allow for the passage of only superfluid $\mathrm{He}$, not normal fluid one. One side of this filter paper $M$ is coated by a thin Au layer $(50 \mathrm{~nm})$. This side is directed towards the arriving Second Sound wave. The second electrode of the capacitor is realized by an immobile brass plate ( $P$ in the figure). The total capacitance of the OST is estimated to be $C_{\mathrm{OST}}=16 \mathrm{pF}$. The OST used here was adapted by DESY [19] following closely the first design of Cornell [9], and supplied to us.

Figure 7 shows the readout circuit for the OST. A $120 \mathrm{~V}$ battery charges the OST. In the first experiments a resistance of $R=(100 \pm 5) \mathrm{k} \Omega$ and a decoupling capacity of $C=(100 \pm 5) \mu \mathrm{F}$ were used, though later these elements were exchanged to optimize the signal amplitude. The best
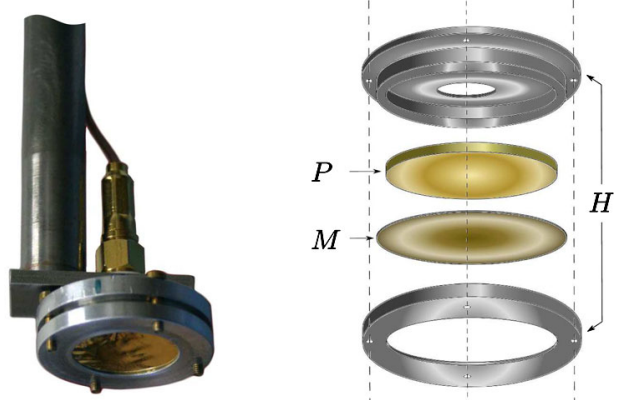

FIG. 6. OST mounted horizontally on the support tube (left) and detail drawing (right) with $H=$ housing, $M=$ porous membrane, and $P=$ immobile brass plate.

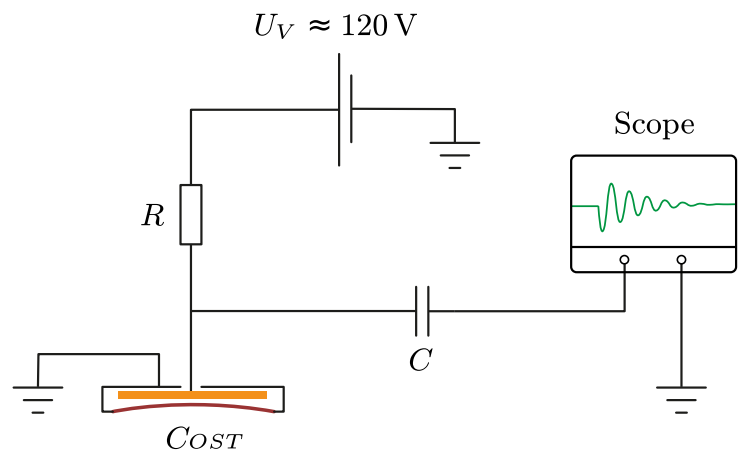

FIG. 7. Readout circuit for the OST. 
signals are obtained with $R=(10 \pm 0.5) \mathrm{M} \Omega$ and $C=$ $(22 \pm 2) \mathrm{pF}$.

The response of the OST to the (arriving) Second Sound wave depends on the density ratio $\rho_{n} / \rho_{s}$. The heat pulse from a quench spot (or from our pointlike heat source) increases $\rho_{n}$, the density of normal fluid He. When the entropy wave reaches the OST, He-II diffuses from the inner volume of the capacitor, the membrane moves inward increasing the capacity $C_{\text {OST }}$. Since the stored charge $Q=$ $C_{\text {OST }} \times U$ is stable, the voltage over the capacitor is lowered, creating a negative pulse on the oscilloscope. In the present experiments, a Tektronix Digital Oszilloskope (series DPO4104) is used in high resolution mode to detect the OST signal with lower noise.

\section{Heat source}

The pointlike heat source is realized by a single $56 \Omega$ SMD resistor with the dimensions $1.5 \times 2.2 \mathrm{~mm}^{2}$. The resistor is placed on a polyvinyl chloride plate. This plate is suspended from three niobium wires (diameter: $0.5 \mathrm{~mm}$ ). Niobium is selected for the wires, as it is superconducting below $9.5 \mathrm{~K}$. Therefore no Ohmic heating occurs, which could be an additional source of Second Sound. Weights are mounted below the plate to damp mechanical oscillations, should they arise. Two of the three wires carry the current pulse to the resistor.

To work under realistic conditions, the power of the heat source should correspond to the heat power $P_{\text {diss }}$ which is dissipated per area $A$ when a quench occurs. According to [5], one can calculate

$$
P_{\text {diss }}=\pi / 2 \times r^{2} \times R_{A} \times H^{2} .
$$

We assume a circular defect of the radius $r=250 \mu \mathrm{m}$. In case of a 9-cell TESLA cavity, operated at a frequency of $1.3 \mathrm{GHz}$, the magnetic field in the equatorial regions is on the order of $H \approx 100$ Oe and the surface resistance $R_{A}$ increases during the quench to a few $\mathrm{m} \Omega$ assuming a quench time of about $5 \mathrm{~ms}$ [8]. Under these assumptions, one estimates a dissipated energy of $0.15 \mathrm{~mJ}$ and a dissipated power of $0.26 \mathrm{~mW}$ [5]. In the experiments different values for voltage and pulse length are used to study the influence of the heat-pulse shape on the OST response (see Fig. 9). At $2 \mathrm{~K}$, the resistance of the heat source is measured to be $R=(82.5 \pm 1.0) \Omega$. A rectangular pulse of $0.2 \mathrm{~ms}$ duration and an amplitude of $4 \mathrm{~V}$, or alternatively a length of $0.8 \mathrm{~ms}$ and an amplitude of $2 \mathrm{~V}$, is applied. Both pulses deliver $(0.039 \pm 0.001) \mathrm{mJ}$. The best response of the OST is found when higher energy is generated in the

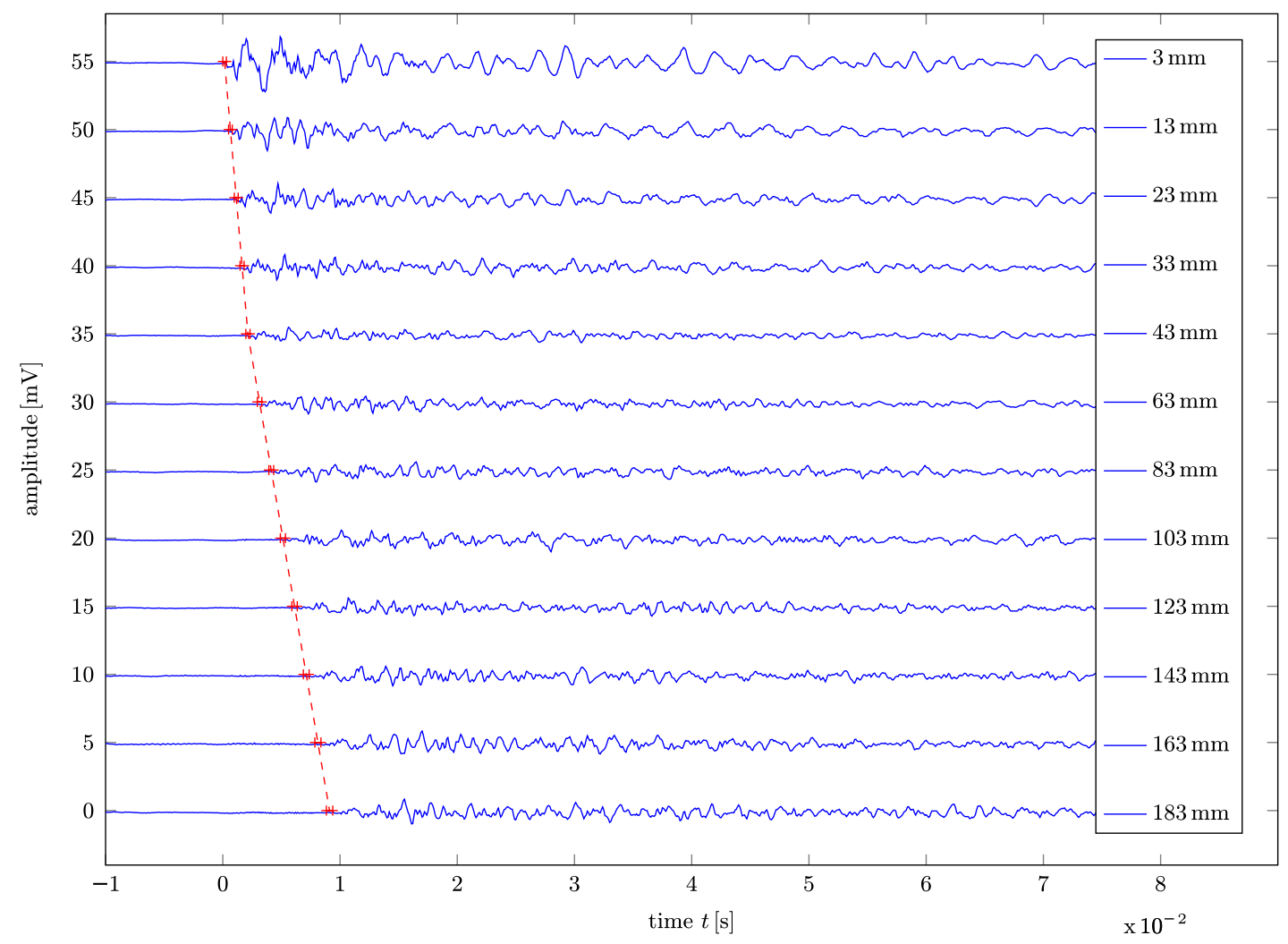

FIG. 8. 12 OST signals recorded in high resolution mode with increasing distance (given on the right side of each signal). The He level was $19.5 \mathrm{~cm}$ above the resistor. The red crosses mark the earliest and latest expected point for the Second Sound to reach the OST; the kink in the red dotted line is due to the change in the step size. All 12 signals are shown with the same scale for the amplitude, from $-5 \mathrm{mV}$ to $+5 \mathrm{mV}$. 


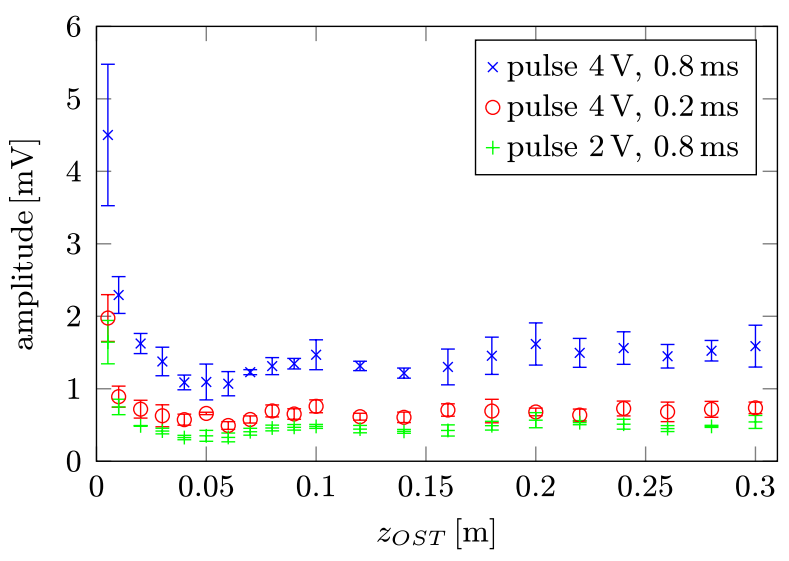

FIG. 9. Signal amplitude at the OST depending on the traveling path $z_{\mathrm{OST}}$ of the Second Sound wave. Three different shapes of the heating pulse are compared.

pulse: with an amplitude of $4 \mathrm{~V}$ and a pulse duration of $0.8 \mathrm{~ms}$, an energy of $(0.156 \pm 0.002) \mathrm{mJ}$ is dissipated.

\section{MEASUREMENTS}

\section{A. Noise suppression}

In the first experiments, a high electronic background of $50 \mathrm{~Hz}$, as well as higher order modes, made it difficult to identify the OST response to pulses of $2 \mathrm{mV}$ amplitude or less. Consequent grounding was used, the application of a battery instead of a variable power supply and the change of the resistor $R$ and the decoupling capacity $C$ (see Fig. 9) increased the signal/noise ratio to some extent. As the next step, the length of the heating pulse was extended to $0.8 \mathrm{~ms}$. The increased heat power caused larger OST amplitudes.

The main source of noise is the rotary vane pump. The OST-sensitive to the First Sound, too-detects $50 \mathrm{~Hz}$ oscillations caused by the running pump. Switching off this pump, one obtains a nearly flat background within $10 \mathrm{~s}$. Nevertheless, switching off the pump has the consequence that the temperature of the liquid He increases, therefore changing the velocity $v_{\text {SeSo }}$. According to [13] and shown in Fig. 3 in the interval from 1.5 to $1.8 \mathrm{~K}$ the velocity $v_{\text {SeSo }}$ is nearly constant with 1.987 to $2.004 \mathrm{~cm} / \mathrm{ms}$, a variation of only $1 \%$ of the minimal value. Therefore, the measurements are performed in this temperature interval which corresponds to a pressure range from 5 to $17 \mathrm{hPa}$. These conditions determine the scheme of the measurements: After a time of about $15 \mathrm{~mm}$, the $\mathrm{He}$ vapor reaches $17 \mathrm{hPa}$. Data taking is stopped, the pump is switched on to reduce the pressure again to $5 \mathrm{hPa}$ within the next 15 min. Then new measurements can be started.

\section{B. Traveling distance variation}

The primary goal is the exact determination of the arrival time of a Second Sound wave. The distance between the heat source and the OST is adjusted in well-defined steps. The zero distance is experimentally determined by mechanically contacting the OST and the heat source. Nevertheless, uncertainties in the distance remain due to the thermal expansion of the used materials. The total error in the distance $z_{\mathrm{OST}}$ (OST/heat source) is estimated to be $\pm 3 \mathrm{~mm}$ [16]. Figure 8 gives an overview of 12 of the signals recorded with increasing distance $z_{\mathrm{OST}}$, indicated on the right axis of the figure.

The heat pulse is released for all 12 spectra at $t=0 \mathrm{~ms}$. Two red crosses are added to each line marking the earliest and latest point in time when the Second Sound signal is supposed to reach the OST, calculated from the actual pressure and distance. The time interval between each pair is the sum of the deviation of the rising and falling edge of the trigger signal and the uncertainty in the Second Sound velocity in the actual temperature interval. The first crosses are connected by a dotted red line. The kink in this line at $z_{\mathrm{OST}}=43 \mathrm{~mm}$ is due to the change in the step size in $z_{\mathrm{OST}}$ from 10 to $20 \mathrm{~mm}$.

Figure 8 shows a complex but reproducible pattern of oscillations, which is different for each selected distance. The signals start with a negative deflection, but with a reduced amplitude. OST signals from the shortest distance $(3 \mathrm{~mm})$ show the highest amplitudes. The amplitudes decrease up to a distance of $40 \mathrm{~mm}$ and then stay at a constant value.

This behavior is clearly seen for the $0.8 \mathrm{~ms}$ long $4 \mathrm{~V}$ heat pulses in Fig. 9 and reflects the fact that the Second Sound propagates as a spherical wave close to the heat source. From a distance of about $40 \mathrm{~mm}$ on, it can approximately be regarded as a planar wave with constant amplitude. The

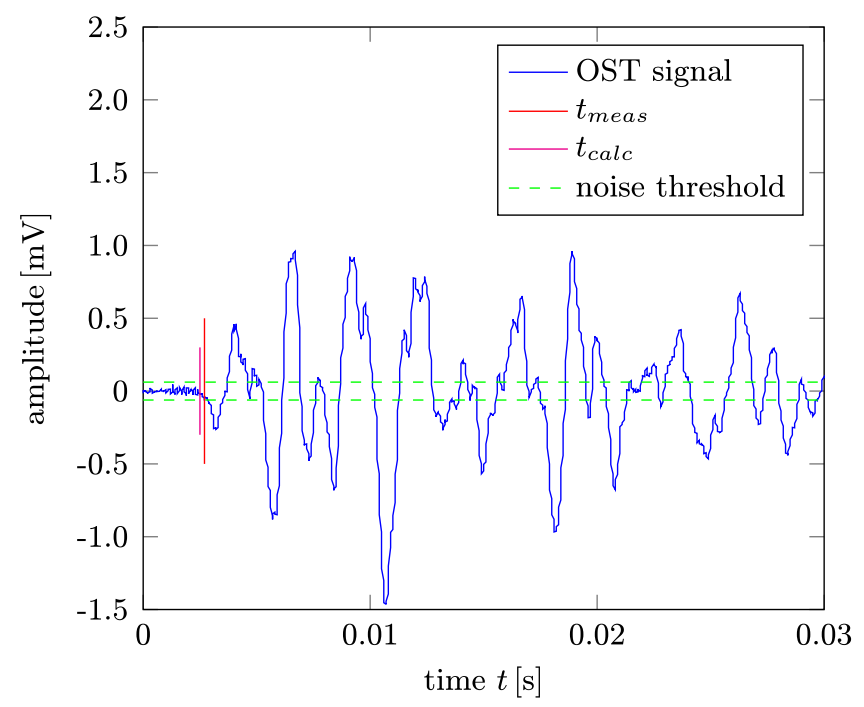

FIG. 10. Starting region of an OST signal. In green the noise band is given, calculated from the measured OST signal prior firing the resistor. The first red line $\left(t_{\text {calc }}\right)$ indicates the position, where the signal is expected theoretically; the second one ( $\left.t_{\text {meas }}\right)$ gives the position where at first the OST signal crosses the noise band. 


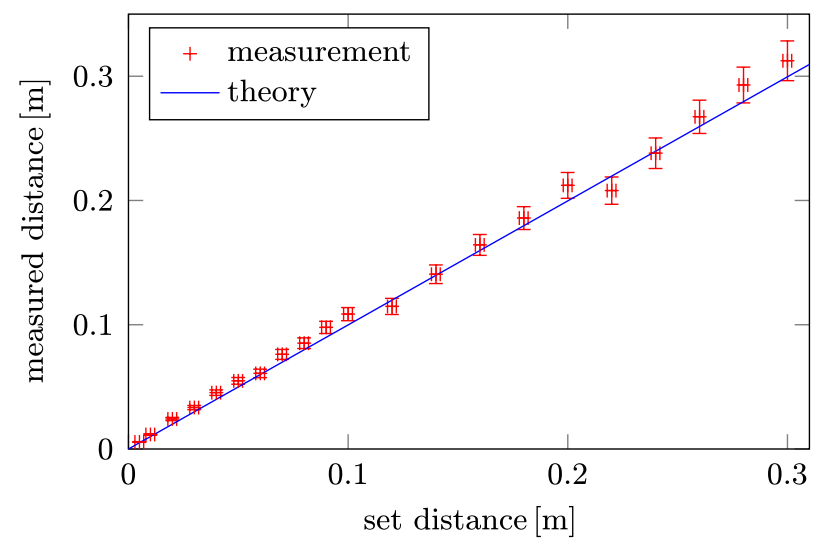

FIG. 11. Distances obtained from data analysis of a full series of OST spectra versus the distance obtained from the mechanical setup.

complicated pattern of the OST response raised the important question on the reproducibility of these patterns. Therefore at each distance $z_{\mathrm{OST}}$ at least 10 OST spectra are recorded under identical conditions. All signal patterns from the same distance are equal, but different for another value of $z_{\mathrm{OST}}$.

As already discussed in Sec. IV B, the OST should respond with a negative pulse to the arrival of a wave front of normal fluid He, i.e., the Second Sound wave from the heat source. The exact determination of this arrival time is crucial for the localization of quench points. As the signal does not start with the full amplitude-a slow onset of membrane oscillations occurs (see Sec. V D) - it is difficult to separate the small negative pulse from the noise background. The maximum amplitude of this noise band can be determined from the data, taken in the time interval prior to the arrival of the Second Sound wave at the OST. The threshold is placed $10 \%$ higher than the maximal noise amplitude (green lines in Fig. 10). The determined arrival time $t_{\text {meas }}$ is indicated together with the arrival time $t_{\text {calc }}$, calculated from the known OST distance and the actual velocity $v_{\mathrm{SeS} o}$. The agreement is satisfactory.

In a full series of OST spectra (obtained with $4 \mathrm{~V}, 0.8 \mathrm{~ms}$ heat pulses) taken at varying $z_{\mathrm{OST}}$, the arrival time $t_{\text {meas }}$ is determined with a computer algorithm. In Fig. 11 the resulting distances (red data points with errors) are plotted versus the set distance $z_{\text {OST }}$. The linear correlation corresponds to the calculated velocity of the Second Sound. The deviation between the measured distance and the set distance $z_{\mathrm{OST}}$ is $6.0 \mathrm{~mm} \mathrm{[20].}$

\section{Reflections}

It is important to test whether reflections of the Second Sound can be observed or not. As a positive consequence, reflections would allow to measure spots without direct sight; on the other hand, they could be the origin of the complex shape of the OST's signal. For this investigation,

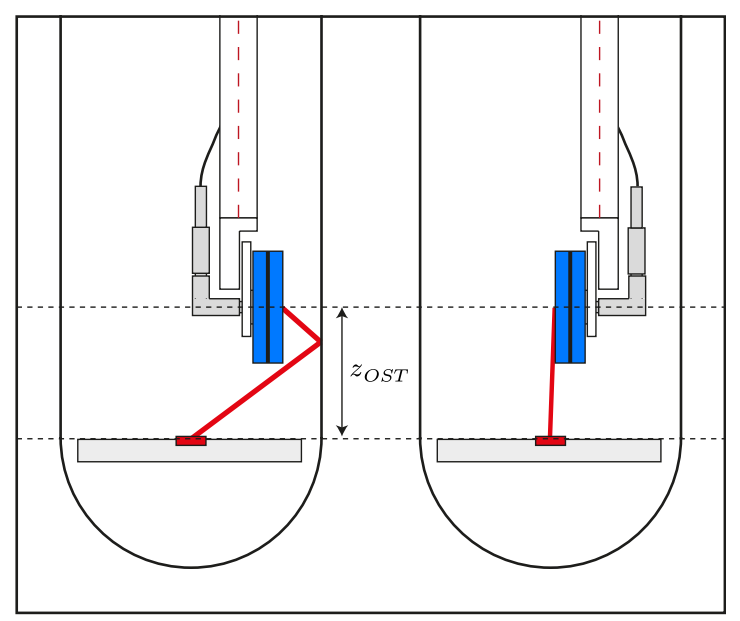

FIG. 12. Drawing of the vertical mounting of the OST. In red are shown the shortest distances for the Second Sound to reach the OST with and without reflection. The heat source is plotted in scale.

the OST is mounted vertically on the supporting rod, which is off axis (see IVA). As shown in Fig. 12 (left), the Second Sound wave can reach the OST only via a reflection on the Dewar glass wall, when the OST is directed towards the wall. A turn of the OST by $180^{\circ}$ (Fig. 12, right) allows for a direct impact of the Second Sound wave on the OST.

The geometry is known, and for the two positions a different signal arrival time at the OST is expected. The result of a series of measurements is shown in Fig. 13. For

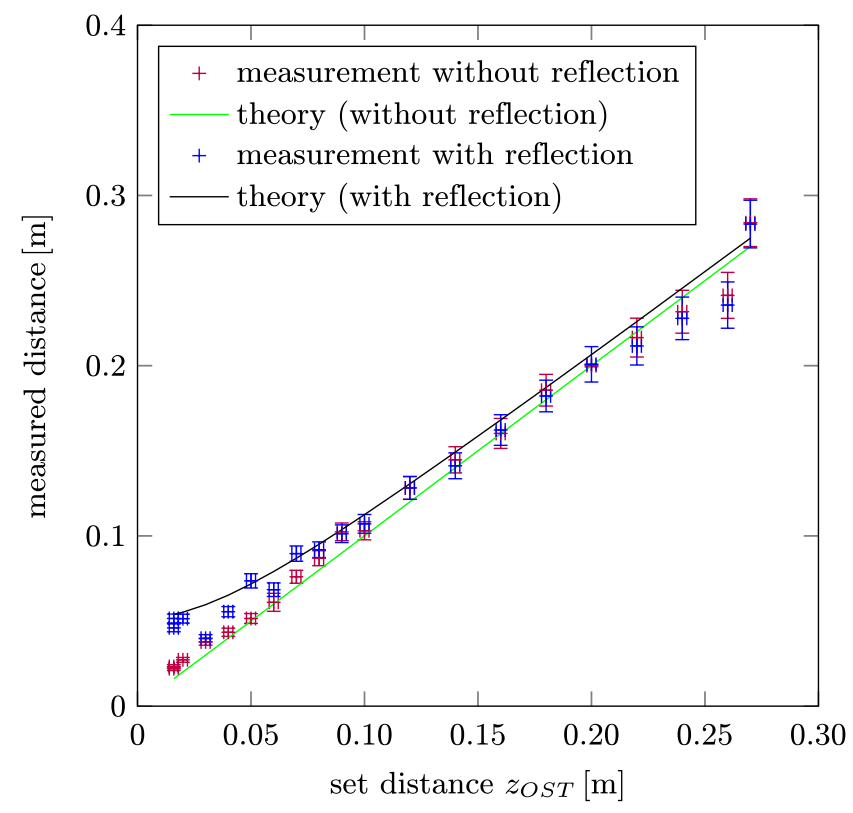

FIG. 13. Without reflection (red crosses) the path length $P_{\text {SoSe }}$ follows the expected linear behavior (see also Fig. 10). Reflected Second Sound (black crosses) has a longer path length $P_{\text {SoSe }}$, according to the distance to the wall. The difference in the path length's $P_{\mathrm{SoSe}}$ shrinks with increasing $z_{\mathrm{OST}}$, as the direct and reflected path length gets more similar. 
19 different values of $z_{\mathrm{OST}}$, the traveling distance of the Second Sound wave is measured for the two OST orientations. All other parameters are held constant (temperature, pressure, height of the He level). The upper bent line in Fig. 13 shows the calculated path length including a reflection. The measured data from the OST facing the wall are in fact larger, and agree with the calculated path length including a reflection. The typical error for the distance is smaller than $10 \mathrm{~mm}$ or $5 \%$, as before [20].

The vertical mounting of the OST gives the possibility to estimate the reflectivity of the wall. The data from the previous experiment are used to compare the signal amplitude from a measurement with and one without reflection. To be independent from any amplitude loss during propagation of the wave, only data with identical traveling distance for both OST orientations (direct impact or after reflection) are compared. This necessitates that $z_{\mathrm{OST}}$ has to be enlarged for the direct impact position. Ten pairs of data are evaluated averaging the amplitude of the first four peaks of the signal for both cases and allow one to estimate a reflection coefficient of $R=0.39 \pm 0.05$ [20].

\section{Oscillations of the OST membrane}

The complex signal shape of the OST (Fig. 8) cannot be explained by reflections inside the Dewar (see V C). Timeresolved Fourier analysis using a Gaussian window is used to study the waveform of the OST signal. Figure 14 shows that three main frequencies contribute to the signal: $f_{1}=(299 \pm 5) \mathrm{Hz}, f_{2}=(553 \pm 6) \mathrm{Hz}$, and $f_{3}=$ $(870 \pm 13) \mathrm{Hz}$ [20]. It can be excluded that these frequencies are caused by the electronics used. Hence, the conclusion is that these are typical oscillations of the OST, most probably of the OST membrane itself.

A spherical, flat membrane can oscillate in many modes which are characterized by certain frequency ratios illustrated in Fig. 15 [21]. The ratio of the three main frequencies from the Fourier analysis is $f_{1}: f_{2}: f_{3}=$ $299: 553: 870=1: 1.85: 2.91$. This can be compared to the theoretical resonant frequencies of a circular membrane. Taking the first modes $01,11,21$, and 12 , we may identify

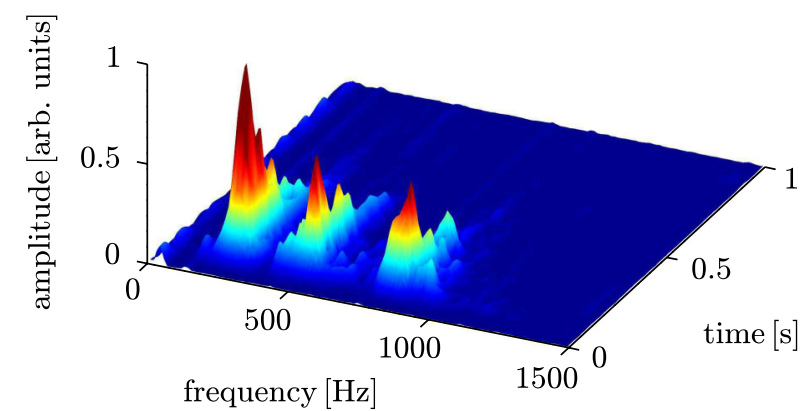

FIG. 14. The 3D diagram shows how the Fourier amplitudes of the three typical frequency distributions decaying in time.

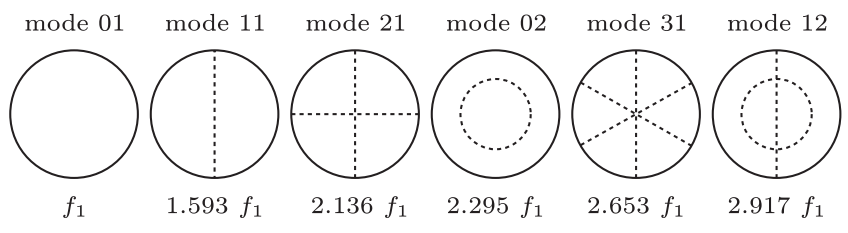

FIG. 15. Graphic representation of the eigenmodes of an oscillating circular membrane with the smallest frequencies; figure adopted according to [7].

$f_{1}$ as the mode 01 and $f_{3}$ as the mode 12. $f_{2}$ shows a value in between the modes 11 and 21. But it cannot be excluded that only a part of the OST membrane is oscillating. Nevertheless, an oscillating membrane explains the shape of the OST response, the long signal and its exponential decay. To obtain further improvement of the quench localization, the OST membrane should be studied in detail, in particular its shape, thickness, and tension.

\section{E. Signal decay time}

The overview (Fig. 8) gives the impression that the OST response in the present setup decays very slowly. The whole range is shown in Fig. 16, with an exponential fit to the absolute amplitudes results in a decay time of $t \approx$ $(0.14 \pm 0.02) \mathrm{s}$. One hypothesis suggests multiple reflections in the small volume of the glass cryostat. But at least 10 reflections are needed to obtain the observed signal length by reflections. The measured reflection coefficient (see V C) excludes this hypothesis, as it would decrease the amplitude down to $1 \%$ of the starting value, whereas the observed value is $1 / e \approx 0.37$. The previous section showed that the OST is a vibratory object which is excited by the incoming Second Sound wavelet. The decay time of the oscillations depends on details of the membranes tension or fixation. OSTs at CERN or DESY have a much shorter signal length [22], although they are fabricated according to the same design.

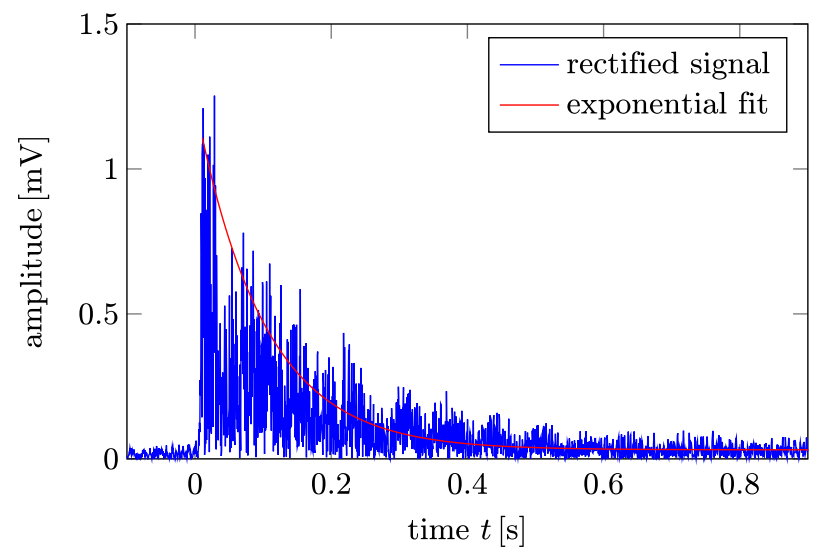

FIG. 16. Exponential decay of the OST signal amplitude (absolute value), measured over a full second. 


\section{CONCLUSIONS}

In the present study we show that the Second Sound wave can still be detected after traveling over a distance of $30 \mathrm{~cm}$. The OST signal can be analyzed to find the exact arrival time of a Second Sound wave, which is the primary means to localize a quench spot. The complex response signal of an OST in the glass He-evaporation cryostat starts with a negative pulse as expected from the working principle. The subsequent oscillations show typical frequencies in certain ratios and an exponential decay of the amplitude; different modes of an oscillating membrane are observed.

Additionally, evidence for reflections of the Second Sound on the walls of the container is found. The question of their influence on the OST's response is important, especially having in mind the complicated shape of a nine-cell TESLA cavity. The experiments demonstrate a high absorption coefficient at the glass walls; one might expect still higher values on a superconducting niobium metal surface. Therefore, Second Sound waves only have a chance to be detected by an OST after one or two reflections. Furthermore, it justifies the use of direct lines of sight as the most basic way to locate the quench spots. In order to increase the sensitivity, the amplitudes of the signal have to be enlarged. This could be done by an amplifier or more detailed knowledge of the oscillating membrane.

\section{ACKNOWLEDGMENTS}

We thank Professor K. Winzer (I. Physikalisches Institut, Georg-August Universität Göttingen) for supplying us with the Dewars for the He-evaporator cryostat and much advice on how to set up the complete cryostat. For valuable discussions about the working principle of an OST, we thank E. Elsen, M. Wenskat, F. Schlander, and $\mathrm{S}$. Aderhold at DESY. The work is supported by the BMBF under Contract No. 05H09PX5/HR5/MG5/RD5

[1] B. Aune, R. Bandelmann, D. Bloess, B. Bonin, A. Bosotti, M. Champion, C. Crawford, G. Deppe, B. Dwersteg, D. A. Edwards, H. T. Edwards, M. Ferrario, M. Fouaidy, P.-D. Gall, A. Gamp, A. Gössel, J. Graber, D. Hubert, M. Hüning, M. Juillard, T. Junquera, H. Kaiser, G. Kreps, M. Kuchnir, R. Lange, M. Leenen, M. Liepe, L. Lilje, A. Matheisen, W.-D. Möller, A. Mosnier, H. Padamsee, C. Pagani, M. Pekeler, H.-B. Peters, O. Peters, D. Proch, K. Rehlich, D. Reschke, H. Safa, T. Schilcher, P. Schmüser, J. Sekutowicz, S. Simrock, W. Singer, M. Tigner, D. Trines, K. Twarowski, G. Weichert, J. Weisend, J. Wojtkiewicz, S. Wolff, and K. Zapfe, Phys. Rev. ST Accel. Beams 3, 092001 (2000).

[2] A. Labanc, TESLA Report No. 1, 2008.
[3] M. Altarelli, R. Brinkmann, M. Chergui, W. Decking, B. Dobson, S. Düsterer, G. Grübel, W. Graeff, H. Graafsma, J. Hajdu, J. Marangos, J. Pflüger, H. Redlin, D. Riley, I. Robinson, J. Rossbach, A. Schwarz, K. Tiedtke, T. Tschentscher, I. Vartaniants, H. Wabnitz, H. Weise, R. Wichmann, K. Witte, A. Wolf, M. Wulff, and M. Yurkov, DESY XFEL Project Group, DESY Report No. 2006-097, 2007.

[4] International Collider Collaboration, International Linear Collider Reference Design Report, Vol. 3: ACCELERATOR, edited by N. Phinney, N. Toge, and N. Walker, 2007.

[5] H.S. Padamsee, J. Knobloch, and T. Hays, RF Superconductivity for Accelerators (Wiley-VCH, Weinheim, Germany, 2008), 2nd ed.

[6] http://www.linearcollider.org/GDE/Director\%27sCorner/2010/17-June-2010-Superconducting-RFcavity-industrialisation.

[7] R.E. Berg and D. G. Stork, The Physics of Sound (Prentice-Hall, Englewood Cliffs, NJ, 1994), 2nd ed.

[8] D. L. Hartill, Cornell Laboratory for Accelerator-Based Sciences and Education, 2008.

[9] Z. A. Conway, D. L. Hartill, H. S. Padamsee, and E. N. Smith, TTC Report No. 06, 2008; Z. A. Conway and D. L. Hartill, in Proceedings of the 23rd Particle Accelerator Conference, Vancouver, British Columbia, Canada (IEEE, Piscataway, NJ, 2009), TU5PFP044.

[10] http://www.chem.queensu.ca/people/faculty/mombourquette/ Chem221/5_PhaseChanges/PhaseDiagrams.asp.

[11] L. Tisza, J. Phys. Radium 1, 164 (1940); 1, 350 (1940); L. D. Landau, J. Phys. USSR 5, 71 (1941); 11, 91 (1947).

[12] C. T. Lane, H. A. Fairbank, and W. M. Fairbank, Phys. Rev. 71, 600 (1947).

[13] O. Chodosh, J. Hiatt, S. Shah, and N. Yan, Department of Physics, Stanford University, 2008.

[14] R. T. Wang, W. T. Wagner, and R. J. Donnelly, J. Low Temp. Phys. 68, 409 (1987).

[15] H. Vennekate, Second Sound as Cavity Diagnostic Tool, 2009, II. Physik-UniGö-Bach2009/04.

[16] B. Schröder, Experiments on second sound using OST and heat spot, 2010, II. Physik-UniGö-Bach2010/08.

[17] H. Preston-Thomas, Metrologia 27, 3, 1990.

[18] Part of the Oerlikon Leybold Vacuum Complete Catalogue, edited by Oerlikon Leybold Vacuum, Chap. 16 (2009).

[19] F. Schlander, S. Aderhold, E. Elsen, and D. Reschke, in Proceedings of Linear Accelerator Conference LINAC2010 (KEK, Tsukuba, Japan, 2011), p. 791.

[20] B. Willenberg, On the Response of an OST to Second Sound, 2011, II. Physik-UniGö-BSc-2011/03.

[21] N.H. Asmar, Partial Differential Equations and Boundary Value Problems with Fourier Series (Pearson Education, Upper Saddle River, NJ, 2005), 2nd ed.

[22] K. Liao, C. Balle, J. Bremer, T. Junginger, W. Wollenberg, W. Weingarten, and H. Vennekate, Second Sound Measurement for SPL Cavity Diagnosis, ILC HiGrade Conf./USA, 2011. 